Open Access

\title{
Diverse origins of hepatitis $C$ virus in HIV co-infected men who have sex with men in Hong Kong
}

Denise P. Chan ${ }^{1}$, Ada W. Lin², Ka Hing Wong ${ }^{2}$, Ngai Sze Wong ${ }^{1}$ and Shui Shan Lee ${ }^{1 *}$

\begin{abstract}
Background: Worldwide, Hepatitis C (HCV) infection has been increasingly recognized in HIV-positive men who have sex with men (MSM). The objective of this study was to characterize the transmission dynamics of acute HCV infection in HIV-positive MSM in Hong Kong using a molecular approach.

Findings: We retrospectively examined 24 HIV-positive MSM with acute HCV infection diagnosed between 2009 and 2014 in Hong Kong. Detection and molecular characterization of HCV was successfully performed in 22 (91.7 \%) patients. Genotype 3a was the most prevalent as identified in 14 (63.6\%) MSM, followed by 1a in 4 (18.2 \%), $6 a$ in 2 $(9.1 \%)$, and 1each (4.5\%) for $1 \mathrm{~b}$ and $2 \mathrm{a}$. The high prevalence of genotype 3a in MSM was in stark contrast to its rarity among HCV infected injection drug users (IDU) in Hong Kong. Phylogenetic analyses revealed a monophyletic HCV-3a cluster composing of MSM without injection history, and a homologous pair with HCV-6a genotype. There was otherwise no temporal or genetic clustering of the corresponding HIV sequences.
\end{abstract}

Conclusions: The origin of sexually acquired acute HCV infections in HIV-positive MSM was diverse and not directly linked with local IDU. The transmission dynamics of HIV and HCV infections in MSM in Hong Kong were evidently unrelated.

Keywords: Acute HCV infection, HIV, Men who have sex with men, Sexual transmission

\section{Findings}

Globally, needle-sharing in injection drug users (IDU) constitutes the main route of hepatitis $\mathrm{C}$ virus (HCV) infection. While sexual transmission of HCV is considered relatively inefficient, it has become increasingly recognized among men who have sex with men (MSM). Since the year 2000, epidemics of acute HCV infection among HIV infected (HIV + ve) MSM in Europe, United States, Australia and Asia have been reported [1-4]. Several cohort studies reported a rising incidence of HCV in HIV + ve MSM following the introduction of HAART, with a pronounced escalation after 2002 [5, 6]. In Hong Kong, the main reservoir of $\mathrm{HCV}$ is the IDU community with an anti-HCV seroprevalence of over $80 \%$ [7]. HIV

\footnotetext{
* Correspondence: sslee@cuhk.edu.hk

Presented in part: 8th IAS Conference on HIV pathogenesis treatment and prevention, Vancouver, Canada 19-22 July 2015 (abstract WEPEC667).

${ }^{1}$ Stanley Ho Centre for Emerging Infectious Diseases, The Chinese University of Hong Kong, Hong Kong, China

Full list of author information is available at the end of the article
}

infection has so far been uncommon in IDU because of the long history and high coverage of methadone maintenance. In view of the rising number of $\mathrm{HCV}$ infections in MSM, yearly screening for $\mathrm{HCV}$ was offered to HIV + ve MSM at the territory's largest HIV clinic which has a caseload of over 2000. To understand the transmission dynamics of acute HCV infection in HIV + ve MSM, a molecular approach was adopted to evaluate the pattern of HIV/HCV co-infection, based on data collected since screening was introduced.

This study was approved by Joint Chinese University of Hong Kong - New Territories East Cluster Clinical Research Ethics Committee. Between August 2009 and March 2014, 24 HIV + ve MSM attending the clinic were diagnosed with a recently-acquired HCV infection. The inclusion criteria for the study were anti-HCV seroconversion and/or the detection of positive $\mathrm{HCV}$ RNA within the preceding 12 months, in the presence of negative anti-HCV status from previous testing. Some infections were picked up because of deranged liver 
Table 1 Characteristics of 24 HIV-positive MSM with sexually acquired acute hepatitis $C$ virus infection in Hong Kong

\begin{tabular}{|c|c|c|}
\hline \multicolumn{3}{|l|}{ Characteristics } \\
\hline $\begin{array}{l}\text { Median age at estimated HCV } \\
\text { infection, years (IQR) }\end{array}$ & 32 & $(27-41)$ \\
\hline \multicolumn{3}{|l|}{ Ethnicity, n (\%) } \\
\hline Chinese & 21 & $(87.5)$ \\
\hline non-Chinese & 3 & $(12.5)$ \\
\hline Time since HIV infection, years (IQR) & 5.0 & $(2.3-8.2)$ \\
\hline On HAART, n (\%) & 23 & $(95.8)$ \\
\hline $\begin{array}{l}\text { Median time from HIV diagnosis } \\
\text { to estimated HCV infection, years (IQR) }\end{array}$ & 3.1 & $(1.2-6.5)$ \\
\hline \multicolumn{3}{|l|}{ HIV subtype $(\mathrm{N}=22)^{\dagger}, \mathrm{n}(\%)$} \\
\hline$B$ & 15 & $(68.2)$ \\
\hline CRF01_AE & 6 & $(27.3)$ \\
\hline CRF07_BC & 1 & $(4.5)$ \\
\hline
\end{tabular}

HAART highly active antiretroviral therapy, HCV hepatitis C virus, IQR interquartile range, $M S M$ men who have sex with men

${ }^{\dagger}$ Of those HCV RNA-positive patients function and a concurrent diagnosis of sexually transmitted infection (STI) like syphilis, suggesting probable venereal exposure. From the available clinical histories, all infections could be linked to recent episodes of unprotected homosexual contacts. The demographics of $\mathrm{HIV} / \mathrm{HCV}$ co-infected MSM in our cohort are shown in Table 1 . The median age at HCV diagnosis was 32 years (interquartile range [IQR]: 27-41 years). All were ethnic Chinese except 2 Caucasians and 1 non-Chinese Asian, and none gave a history of drug injection. The median interval between HIV diagnosis and HCV seroconversion was 3.1 years (IQR: 1.2-6.5 years). One MSM was diagnosed with HCV infection within 6 months of his HIV diagnosis. The HIV-1 subtype was confirmed in 22 patients: subtype B in 15 (68.2\%), CRF01_AE in 6 (27.3\%) and CRF07_BC in 1 (4.5\%). A majority of the HCV infections diagnosed in these HIV + ve MSM after 2012 belonged to genotype 3a (14/16, 87.5 \%) (Fig. 1).

Molecular characterization of $\mathrm{HCV}$ was performed as previously described [8]. Briefly, a fragment of the HCV NS5B region was amplified by reverse-transcriptase nested PCR. Amplification and sequencing of the NS5B fragment was successful in 22 samples $(91.7 \%)$, with the remaining 2 (8.3 \%) having undetectable HCV RNA. A neighbor-joining phylogenetic tree was constructed with

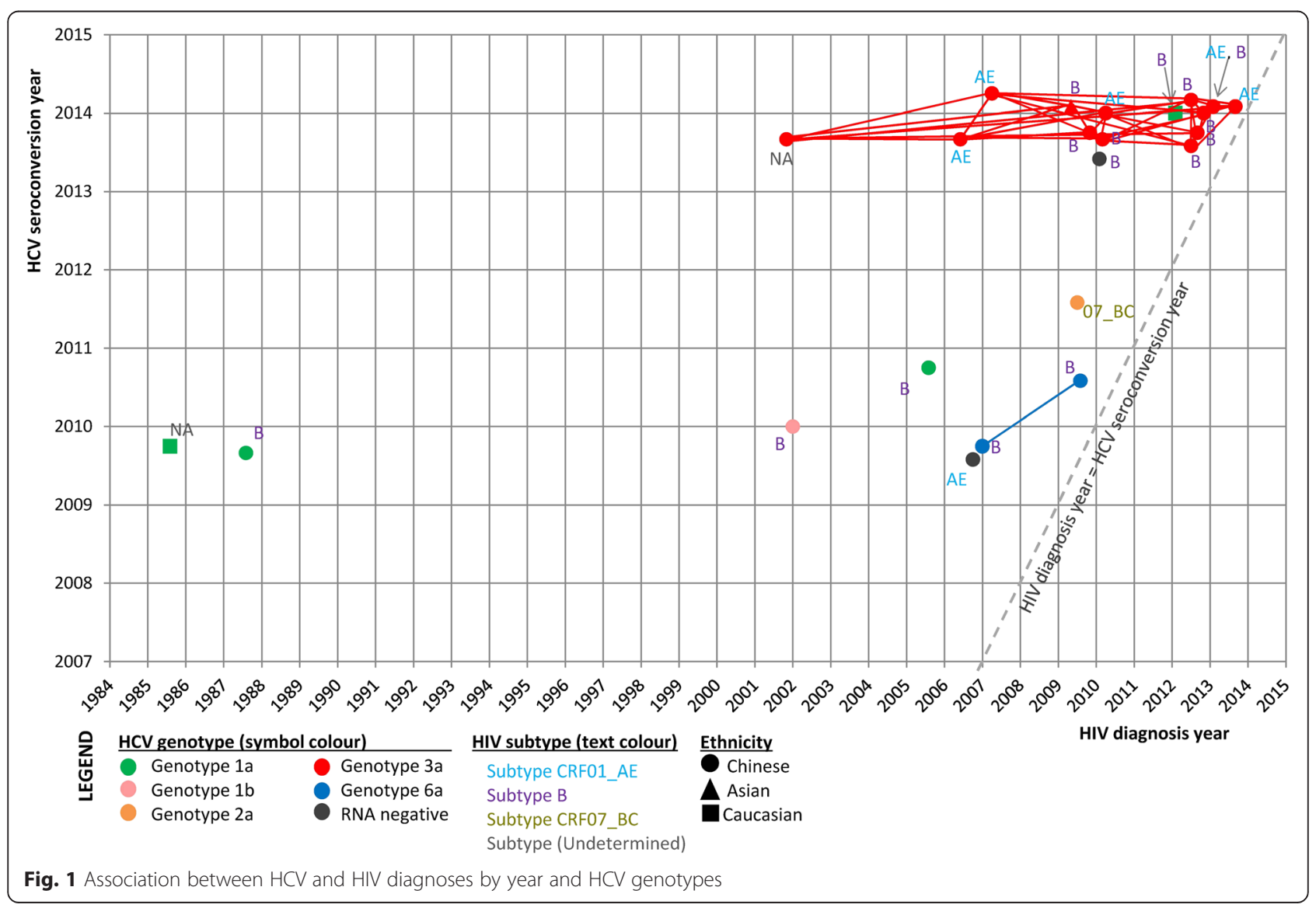


Kimura-2 parameter model using the MEGA6.0 software (http://www.megasoftware.net). Reference strains for HCV genotyping and worldwide NS5B sequences for phylogenetic analyses were retrieved from GenBank. Overall, the genotypic identity of $\mathrm{HCV}$ was diverse. A majority belonged to genotype $3 \mathrm{a}(\mathrm{n}=14 ; 63.6 \%)$ followed by $1 \mathrm{a}$ $(\mathrm{n}=4 ; 18.2 \%) ; 6 \mathrm{a}(\mathrm{n}=2 ; 9.1 \%) ; 1 \mathrm{~b}(\mathrm{n}=1 ; 4.5 \%)$; and $2 \mathrm{a}$ $(\mathrm{n}=1 ; 4.5 \%)$. Phylogenetic analysis suggested that there were a total of 8 circulating viruses, of which 2 showed significant clustering. One was a monophyletic HCV-3a cluster (bootstrap value $>70 \%$ ), while the other was a HCV-6a pair (Fig. 2a). Members of the HCV-3a cluster were all diagnosed between 2013 and 2014. Despite the clustered $\mathrm{HCV}$ sequences, their corresponding HIV sequences did not show any genetic or temporal clustering. These samples contained HIV-1 of 2 subtypes: CRF01_AE $(n=5)$ and B $(\mathrm{n}=8)$, as determined from pol gene sequences obtained for genotypic resistance testing [9] (Fig. 2b).

Figure 3a shows the results of phylogenetic analysis for the HCV genotype 3a samples. The HCV-3a sequences were distinctly different from those obtained from IDU in Hong Kong and Mainland China. The considerably shorter genetic distance between sequences within the HCV-3a cluster and their low genetic diversity, with diagnosis made within 2 years, suggested the occurrence of rapid virus spread in the HIV + ve MSM community, which might have resulted from a single source of recent introduction within Hong Kong. The origin of this cluster could not be further characterized as no similar sequences in Hong Kong could be found. HCV transmission might have arisen from the spillover of the virus from IDU to an MSM long time ago, as the sequences did not show any similarity with

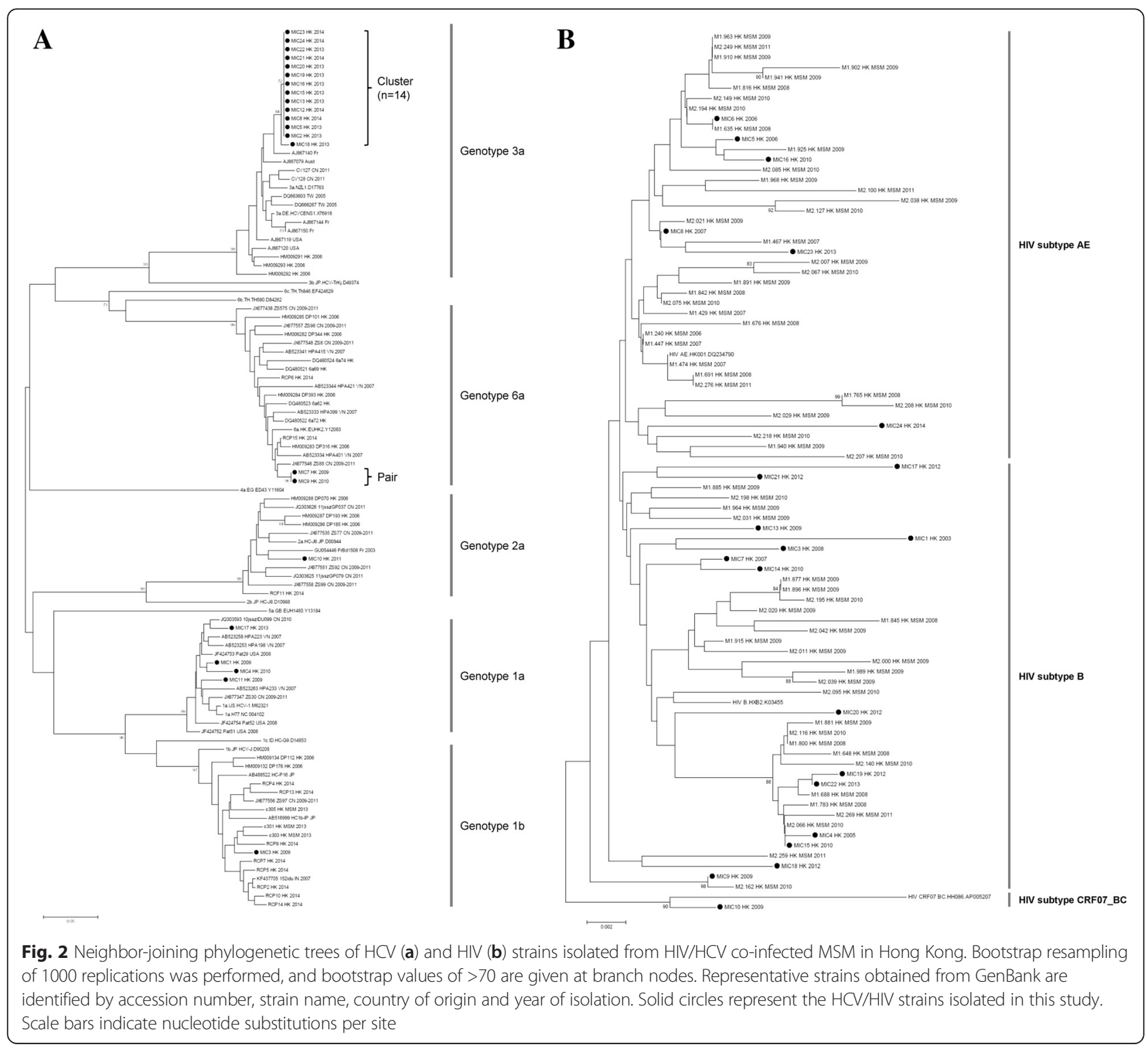




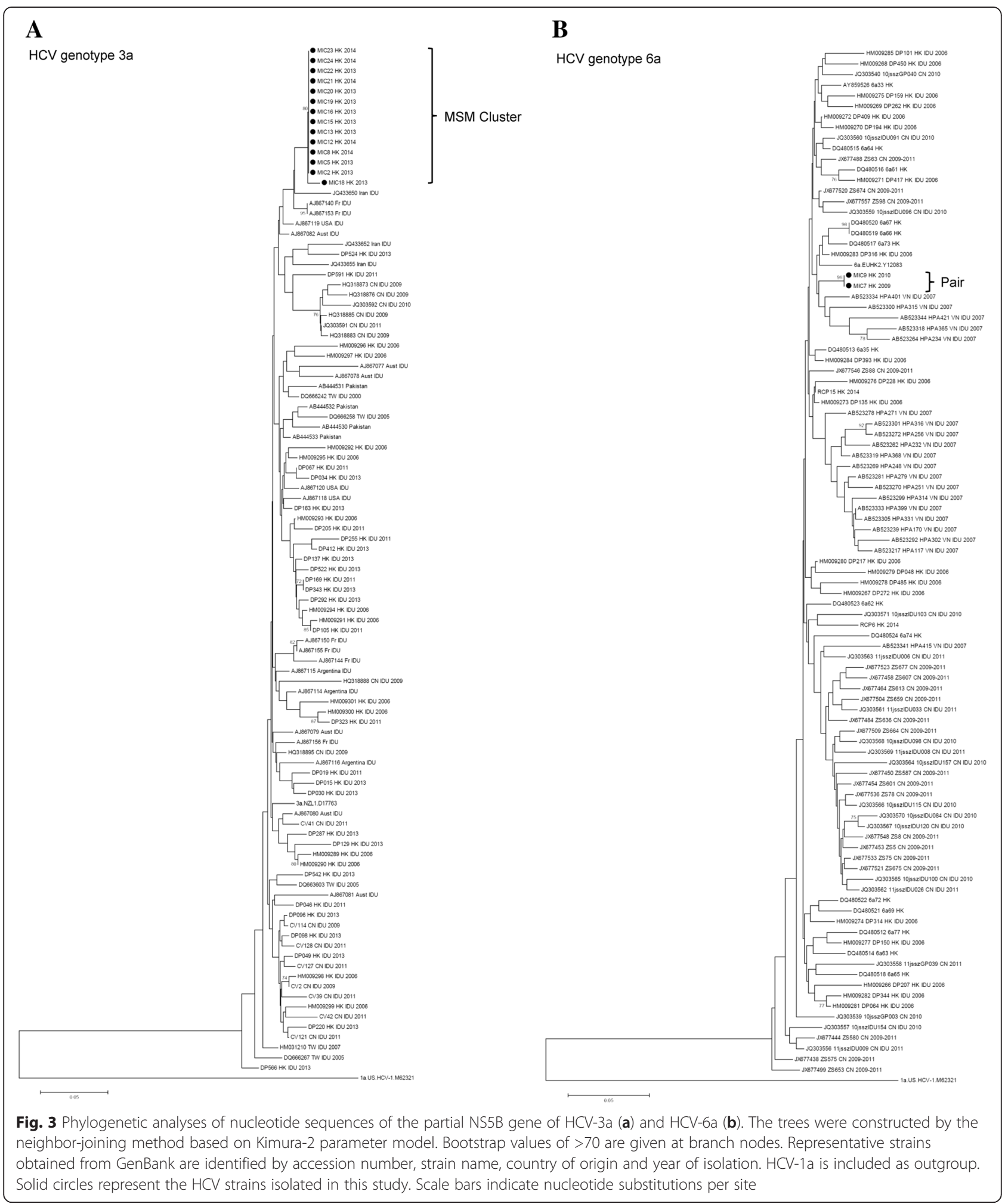

those in China, Southeast Asia or Australia. In Australia, genotype $3 \mathrm{a}$ actually constituted one of the most prevalent virus strains in acute $\mathrm{HCV}$ infections. Unlike the situation in Hong Kong, however, overlapping and coexistence of
HCV-3a clusters between the IDU and HIV + ve MSM populations could be demonstrated in Australia [10].

Apart from the genotype 3 a cluster, a homologous pair of near-identical genotype 6a sequences was identified, 
the diagnoses of which were made earlier in 2009 and 2010 before emergence of the HCV-3a cluster, with corresponding HIV diagnoses in 2006 and 2009. Further investigation revealed that the 2 persons were not known to one another. It is possible that the 2 near-identical virus strains formed part of a bigger but yet unidentified cluster, which could be circulating in HIV-ve MSM in Hong Kong. Alternatively, transmission might have ceased to occur after the diagnosis of the latest case in 2010. Phylogenetic analysis suggested that the HCV-6a sequence in the MSM pair was more similar to local IDU than those in Mainland China (Fig. 3b). Thus the virus might have been transmitted from a local MSM who injected drug, or through a sex partner who was an $\mathrm{HCV}$ infected IDU. The remaining $6 \mathrm{HCV}$ viruses belonged to genotypes $1 \mathrm{a}, 1 \mathrm{~b}$ and $2 \mathrm{a}$. Globally, genotype 1 accounts for around $46 \%$ of all HCV infections and is the most widely circulating genotype in Europe and North America [11]. Four of the viruses in our cohort belonged to this genotype, but all existed as singletons without evidence of clustering, and 2 were isolated from Caucasians. It is possible that these infections were acquired outside Hong Kong, or that local transmission has not occurred to a significant extent. The remaining HCV-2a infection, diagnosed in 2011, occurred in an HIV + ve MSM infected with HIV CRF07_BC, a signature HIV-1 subtype which originated in IDU in Yunnan with subsequent spread to Xinjiang and other provinces [12]. Apparently, local dissemination had not occurred for any of the non-3a HCV viruses after the diagnoses of these infections before 2012.

In conclusion, similar to the situation in Western countries, $\mathrm{HCV}$ has emerged as a sexually acquired infection in HIV + ve MSM in Hong Kong in the last decade. Phylogenetic investigations showed that 8 different $\mathrm{HCV}$ viruses were implicated in this new epidemic, of which one (HCV-3a) displayed significant genetic and temporal clustering. The $\mathrm{HCV}$ infections in HIV + ve MSM in Hong Kong did not form part of the reported international MSM-specific $\mathrm{HCV}$ transmission networks composing of HCV-1a and HCV-4d genotypes [13]. The discovery of a HCV-6a pair suggested that some of the infections might have arisen from local IDU. Evidently, the transmission of HIV and HCV in MSM in Hong Kong had differed both in onset time and their phylogenetic relationship. Apparently, HCV has spread in different MSM networks that became established after HIV infection. Instead of global dissemination in the MSM populations, HCV had probably come from local or nearby nonMSM sources, with multiple introductions at different time points. As sexual transmission of $\mathrm{HCV}$ is generally believed to be inefficient, its emergence in HIV + ve MSM populations could have resulted from an interlay of high risk sexual behaviors, host susceptibility and viral factors which have yet to be identified.

\section{Abbreviations}

HCV: Hepatitis C virus; HIV + ve: HIV infected; IDU: Injection drug users; IQR: Interquartile range; MSM: Men who have sex with men.

\section{Authors' contributions}

DC developed the study protocol and drafted the manuscript. AL oversaw data collection. KHW contributed to reviewing the manuscript. NSW analyzed and interpreted the results. SSL conceptualized the study and participated in critical review of the revised manuscript. All authors had read and approved the final manuscript.

\section{Acknowledgments}

This work was supported by the Health and Medical Research Fund (Project reference no: CUHK-Portfolio-C6) of Food and Health Bureau of the Hong Kong Special Administrative Region Government. The authors acknowledge the technical support of Li Ka Shing Institute of Health Sciences, The Chinese University of Hong Kong.

\section{Competing interests}

The authors declare that they have no competing interests.

\section{Disclaimers}

The opinions expressed by the authors contributing to this journal do not necessarily reflect the opinions of the Centre for Health Protection of Department of Health in Hong Kong with which some authors are affiliated.

\section{Author details}

'Stanley Ho Centre for Emerging Infectious Diseases, The Chinese University of Hong Kong, Hong Kong, China. ${ }^{2}$ Centre for Health Protection, Department of Health, Hong Kong, China.

Received: 3 February 2015 Accepted: 30 July 2015

Published online: 08 August 2015

\section{References}

1. Browne R, Asboe D, Gilleece Y, Atkins M, Mandalia S, Gazzard B, et al. Increased numbers of acute hepatitis $C$ infections in HIV positive homosexual men; is sexual transmission feeding the increase? Sex Transm Infect. 2004;80:326-7.

2. Luetkemeyer A, Hare CB, Stansell J, Tien PC, Charlesbois E, Lum P, et al Clinical presentation and course of acute hepatitis $C$ infection in HIV-infected patients. J Acquir Immune Defic Syndr. 2006;41:31-6.

3. Matthews GV, Hellard M, Kaldor J, Lloyd A, Dore GJ. Further evidence of HCV sexual transmission among HIV-positive men who have sex with men: response to Danta et al. AIDS. 2007;21:2112-3.

4. Su YC, Liu WC, Chang LH, Wu PY, Luo YZ, Wu CH, et al. Incidence of recent HCV infection among persons seeking voluntary counselling and testing for HIV and sexually transmitted infections in Taiwan. J Int AIDS Soc. 2014;17 (4 Suppl 3):19640.

5. van de Laar TJ, Matthews GV, Prins M, Danta M. Acute hepatitis C in HIV-infected men who have sex with men: an emerging sexually transmitted infection. AIDS. 2010;24:1799-812.

6. van der Helm JJ, Prins M, del Amo J, Bucher HC, Chene G, Dorrucci M, et al. The hepatitis C epidemic among HIV-positive MSM: incidence estimates from 1990 to 2007. AIDS. 2011;25:1083-91.

7. Wong NS, Chan PC, Lee SS, Lee SL, Lee CK. A multilevel approach for assessing the variability of hepatitis $C$ prevalence in injection drug users by their gathering places. Int J Infect Dis. 2013;17:e193-8.

8. Chan DP, Lee SS, Lee KC. The effects of widespread methadone treatment on the molecular epidemiology of hepatitis $C$ virus infection among injection drug users in Hong Kong. J Med Virol. 2011:83:1187-94.

9. Chen JH, Wong KH, Li PC, Chan KK, Lee MP, To SW, et al. In-house human immunodeficiency virus-1 genotype resistance testing to determine highly active antiretroviral therapy resistance mutations in Hong Kong. Hong Kong Med J. 2012;18:20-4.

10. Matthews GV, Pham ST, Hellard M, Grebely J, Zhang L, Oon A, et al. Patterns and characteristics of hepatitis $C$ transmission clusters among HIV-positive 
and HIV-negative individuals in the Australian trial in acute hepatitis C. Clin Infect Dis. 2011;52:803-11.

11. Gower E, Estes C, Blach S, Razavi-Shearer K, Razavi H. Global epidemiology and genotype distribution of the hepatitis $\mathrm{C}$ virus infection. J Hepatol. 2014;61:S45-57.

12. Meng Z, Xin R, Zhong P, Zhang C, Abubakar YF, Li J, et al. A new migration map of HIV-1 CRF07_BC in China: analysis of sequences from 12 provinces over a decade. PLoS One. 2012;7, e52373.

13. van de Laar T, Pybus O, Bruisten S, Brown D, Nelson M, Bhagani S, et al. Evidence of a large, international network of HCV transmission in

HIV-positive men who have sex with men. Gastroenterology. 2009;136:1609-17.

\section{Submit your next manuscript to BioMed Central} and take full advantage of:

- Convenient online submission

- Thorough peer review

- No space constraints or color figure charges

- Immediate publication on acceptance

- Inclusion in PubMed, CAS, Scopus and Google Scholar

- Research which is freely available for redistribution 